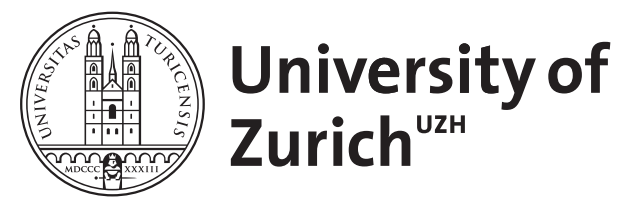

\title{
On Josephson effects in insulating spin systems
}

\author{
Schilling, Andreas ; Grundmann, Henrik
}

\begin{abstract}
We discuss an experiment in which two magnetic insulators that both show a field-induced magnetic ordering transition are weakly coupled to one another and are placed into an external magnetic field. If the respective magnetic states can be interpreted as phase-coherent Bose-Einstein condensates of magnetic bosonic quasiparticles, one expects the occurrence of Josephson effects. For two identical systems, the resulting d.c. Josephson effect formally represents a constant quasiparticle Josephson current across the weak link, which turns out to be unobservable in an experiment. For magnetic insulators with different critical fields, a spontaneous alternating quasiparticle current develops with a leading oscillation frequency View the MathML source that is determined by the difference between the critical fields. As a result of the coupling, additional sidebands appear in the energy spectrum of the coupled device that would be absent without phase coherence. We discuss the primary conditions for such an effect to take place and conclude that its detection can be feasible for a proper choice of compounds with suitable and realistic material parameters.
\end{abstract}

DOI: https://doi.org/10.1016/j.aop.2012.04.004

Posted at the Zurich Open Repository and Archive, University of Zurich

ZORA URL: https://doi.org/10.5167/uzh-72328

Journal Article

Accepted Version

Originally published at:

Schilling, Andreas; Grundmann, Henrik (2012). On Josephson effects in insulating spin systems. Annals of Physics, 327(9):2301-2309.

DOI: https://doi.org/10.1016/j.aop.2012.04.004 


\title{
On Josephson effects in insulating spin systems
}

\author{
Andreas Schillinga,*, Henrik Grundmann ${ }^{\mathrm{a}}$ \\ ${ }^{a}$ Physics Institute, University of Zürich, Winterthurerstrasse 190, CH-8057 Zürich
}

\begin{abstract}
We discuss an experiment in which two magnetic insulators that both show a field-induced magnetic-ordering transition are weakly coupled to one another and are placed into an external magnetic field. If the respective magnetic states can be interpreted as phase coherent Bose-Einstein condensates of magnetic bosonic quasiparticles, one expects the occurrence of Josephson effects. For two identical systems, the resulting d.c. Josephson effect formally represents a constant quasiparticle Josephson current across the weak link, which turns out to be unobservable in an experiment. For magnetic insulators with different critical fields, a spontaneous alternating quasiparticle current develops with a leading oscillation frequency $\omega_{\text {a.c. }}$ that is determined by the difference between the critical fields. As a result of the coupling, additional sidebands appear in the energy spectrum of the coupled device that would be absent without phase coherence. We discuss the primary conditions for such an effect to take place and conclude that its detection can be feasible for a proper choice of compounds with suitable and realistic material parameters.
\end{abstract}

Keywords: Tunneling, Josephson effect, Quantized spin models, Macroscopic quantum phenomena in magnetic systems, Josephson devices

PACS: 03.75.Lm, 75.10.Jm, 75.45.+j, 85.25.Cp

\section{Introduction}

Quantum spin systems in solids have been a subject of intensive research, both theoretically and experimentally. A number of such systems show magnetic-field induced phase transitions at zero temperature that have been interpreted as a Bose-Einstein condensation of magnetic bosonic quasiparticles [1-8].

In insulating dimerized spin-1/2 systems, for example, one expects a condensation of triplet bosonic quasiparticles ("triplons") above a certain critical field $H_{c}[4,5]$ where the energy difference between the ground-state singlet and the lowest excited triplet states vanishes due to the Zeeman splitting. In the language of magnetism, this condensation corresponds to a field-induced antiferromagnetic ordering that is associated with the appearance

\footnotetext{
*Corresponding author

Email address: aschilling@physik.uzh.ch (Andreas Schilling)
} 
of a staggered magnetization $[1,3,4]$. However, the stability of such a condensate $[9,10]$ and even the applicability of the BEC concept [11-13] have been questioned.

Already known Bose-Einstein condensates (BEC) include superfluid helium [14, 15], dilute atomic gas clouds [16] and pumped exciton-polariton and magnon condensates [17-20]. Several experimental proofs for the existence of a macroscopic quantum state have been reported, e.g., from interference experiments [21-23], from the observation of vortices [24-26] and from Josephson effects [27-32], and even superfluid properties can be ascribed to most of these systems $[14,15,33-36]$. Such hallmarks of a true BEC state are all based on the existence of a macroscopic phase-coherent state, but none of these has ever been observed in any insulating quantum spin system.

In this article we propose an experiment to probe the macroscopic phase coherence in insulating spin systems by observing the a.c. Josephson effect across a weak link. A successful detection of this effect would represent a direct proof for the existence of a macroscopic quantum state. We start with a discussion about the macroscopic wavefunction and the chemical potential of a triplon BEC in a magnetic insulator at zero temperature (sections 2 and 3). We then consider the Josephson equations for a system of two weakly coupled triplon BECs (section 4) and show how the a.c. Josephson effect can be identified in principle. In section 5 we discuss several constraints for a system with realistic material parameters, and we finally conclude under realistic assumptions that such an experiment is indeed feasible.

\section{The macroscopic wavefunction of a triplon BEC}

The amplitude of the macroscopic wavefunction $\psi=\sqrt{n} e^{i \phi}$ describing a Bose-Einstein condensate of $N$ triplons in a magnetic insulator at zero temperature is related to the density $n$, here defined as $n=N / N_{d}$ (with $N_{d}$ the total number of dimers) so that $0 \leq n \leq 1$. To first approximation and near the critical field $H_{c}$, this density is proportional to the longitudinal magnetic moment $M_{z}=g \mu_{B} n N_{d}=g \mu_{B} N[5,8]$, where $g$ is the Landé $g$-factor and $\mu_{B}$ the Bohr magneton. As the equilibrium $M_{z}$ is a well-defined quantity for a fixed value of the external magnetic field $H$ in the thermodynamic limit, the triplon number $N=M_{z} / g \mu_{B}$ can be considered to be conserved.

The phase $\phi$ of the macroscopic wavefunction in magnetic insulators is closely related to (but not identical with) the angle $\varphi$ of the transverse magnetic moments within the plane perpendicular to the main magnetic field $H$ [7, 37, 38]. A plausibility argument for this fact is that the particle number $N$ is canonically conjugate to the phase $\phi$ of the macroscopic wavefunction on the one hand, but also (via the proportionality of $N$ to the longitudinal magnetic moment $M_{z}$ and therefore to the associated component of the angular momentum $L_{z}$ parallel to $H$ ) to the angle variable $\varphi$ in the plane perpendicular to $H$ on the other hand. In contrast to the time dependent global phase $\phi(t)$ of the macroscopic wavefunction, the angle $\varphi$ between the transverse magnetic moments and the frame of reference given by the crystal lattice must be constant in time, because the Landau-Lifshitz-Gilbert equation does not allow for a perpetual spin precession of the staggered magnetization in the magnetic ground state. This apparent discrepancy can be easily resolved if one interprets the global phase of the macroscopic wavefunction as $\phi(t)=\varphi-\omega t$ (with $\omega$ provided by the time- 
dependent Schrödinger equation), i.e., the in-plane angle $\varphi$ of the staggered magnetization corresponds to an undetermined phase constant $\phi_{0}$. This interpretation of $\phi$ and $\varphi$ ensures that the expectation values of $L_{z}$ and of the energy $E$ per triplon are $\left\langle L_{z}\right\rangle_{\psi}=\hbar$ and $\langle E\rangle_{\psi}=\hbar \omega$, respectively.

In an axially symmetric spin system, $\varphi$ must be able to take any arbitrary constant value between 0 and $2 \pi$ [37], which is to some extent in contrast to experimental observations where $\varphi$ seems to be locked to a material-specific value $[39,40]$. We will address this issue of a violated axial symmetry later on in section 5.2. In the following we shall focus on the time evolution of the global phase $\phi(t)$ of the macroscopic wavefunction because its relation to the angle $\varphi$ does not play any further important role here.

\section{The chemical potential $\mu$}

A decisive prerequisite for an a.c. Josephson effect to take place between two BECs is the presence of a steady non-zero difference $\Delta \mu$ between the respective chemical potentials. In a condensate of magnetic quasiparticles in an insulating spin system, this chemical potential is usually taken as $\mu=g \mu_{B} \mu_{0}\left(H-H_{c}\right)[1,2,4,5]$. As in all BECs of weakly interacting light bosons in the limit $n \ll 1$, a non-zero $\mu$ is related to $n$ according to $n=\mu / v_{0}$. The interaction constant $v_{0}$ describes the repulsive hard-core interaction between the bosonic quasiparticles, and is in magnetic insulators determined by the finite inter-dimer interactions which result in the formation of dispersive energy bands of the triplet excitations [4]. It is important to emphasize that the appearance of a non-zero value of $\mu=\partial E / \partial N$ (with $E$ the total energy of the condensate) is common to all interacting BECs that are treated within the Gross-Pitaevskii formalism [41, 42], and is not restricted to the magnetic systems under study here.

In the following we aim to bring two of such condensates into contact in order to investigate possible analogues to the d.c. and a.c. Josephson effects. Therefore it is indispensable to examine whether or not the above definition of the chemical potential $\mu$ is actually applicable for treating such an experiment correctly. If we consider, for example, two different magnetic insulators $\left(\alpha\right.$ and $\beta$ ) with different critical magnetic fields $H_{c, \alpha}$ and $H_{c, \beta}$ that are placed into a common external magnetic field $H$, an apparently constant difference $\Delta \mu=\mu_{\alpha}-\mu_{\beta}=g \mu_{0} \mu_{B}\left(H_{c, \beta}-H_{c, \alpha}\right)$ is maintained by the external magnetic field (see Fig. $1)$. This situation is formally equivalent to that of a device composed of two pieces of the same material with a single $H_{c}$, but placed into two different magnetic fields differing by $\Delta H$. As we shall see later on, the necessary field gradient for the latter type of experiment to be successful is of the order of $\mu_{0} \Delta H \approx 0.5 \mathrm{~T}$ along a length of $\approx 0.5 \mathrm{~nm}$. This is technically out of reach, and we therefore do not consider this scenario any further.

This non-zero difference $\Delta \mu$ is not simply a result of choosing different reference points of the energy scale for the two condensates. Firstly, the difference in the critical fields $H_{c, j}$ $(j=\alpha$ or $\beta$ ) originally stems from a difference in microscopic inter-dimer and intra-dimer coupling constants that determine the individual energy gaps, i.e. the energy separation of the respective ground-state singlet and the triplet states in zero magnetic field. To close these energy gaps, different external magnetic fields $H_{c, j}$ must be applied beyond which 

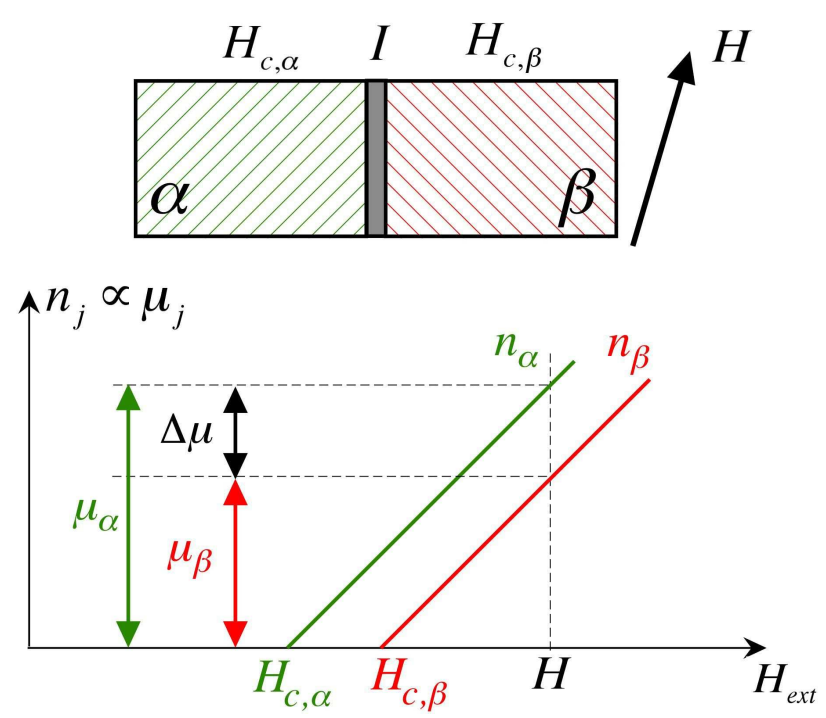

Figure 1: Sketch of an experiment in which two magnetic insulators with different critical fields $H_{c, j}(j=$ $\alpha, \beta)$ are weakly coupled to one another through a magnetically inert spacer layer $I$ and are placed into an external magnetic field $H>H_{c, j}$. The respective condensate densities $n_{j}$ and the chemical potentials $\mu_{j}$ vary approximately linearly with $H$.

the respective ground-state triplons condense. On an absolute energy scale (with the zero point chosen for a state with isolated spin- $1 / 2$ particles in vacuum and in zero magnetic field), the condensation occurs at the energy level of the respective singlet states, i.e., at $E_{j}=-3 J_{j} / 4$ per dimer, where the $J_{j}$ denote the respective intra-dimer coupling energies. Secondly and most importantly, the relevant quantity that enters the problem of treating the Josephson effect is the relative energy gain (or loss) $\partial E / \partial N$ upon creation (or annihilation) of one triplon quasiparticle to (or from) the condensate, from (or into) the singlet sea, which is actually $n_{j} v_{0, j}=\mu_{j}=g_{j} \mu_{B} \mu_{0}\left(H-H_{c, j}\right)$ per dimer ${ }^{1}[3-5,7]$. Therefore, the $\mu_{j}$ (and along with them the condensate densities $\left.n_{j}=\mu_{j} / v_{0, j}\right)$ are indeed different in a common external magnetic field that exceeds both $H_{c, \alpha}$ and $H_{c, \beta}$ (see Fig. 1), and $\Delta \mu$ can then be considered as the analogue to an external voltage controlling the difference between the chemical potentials in a superconducting Josephson junction.

\section{Josephson effects}

\subsection{The Josephson equations}

We now apply this concept to a system of two dimerized spin systems at zero temperature and with different critical fields $H_{c, \beta}>H_{c, \alpha}$ beyond which the respective magnetic quasiparticles are supposed to condense. We initially assume a perfect axial symmetry of

\footnotetext{
${ }^{1}$ This result for $\mu$ can be illustrated by identifying $E$ with the energy of the magnetized system in a field $H, E=\mu_{0} \int_{H_{c}}^{H} M_{z}\left(H^{\prime}\right) \mathrm{d} H^{\prime}$ with $M_{z}=g \mu_{B} N$ and $N=g \mu_{B} \mu_{0}\left(H-H_{c}\right) N_{d} / v_{0}$, so that $E(N)=N^{2} v_{0} / 2 N_{d}$ and $\mu=\partial E / \partial N=n v_{0}$.
} 
the two magnetic systems with respect to the direction of the external magnetic field, but we will discuss the case of violated axial symmetry later on in section 5.2. The boundary layers of the two systems are assumed to be weakly coupled to one another, and we place the device into a magnetic field $H>H_{c, j}$ (see Fig. 1). For simplicity, we also assume $v_{0, \alpha} \approx v_{0, \beta}=v_{0}$ and $g_{\alpha} \approx g_{\beta}=g$, which is not essential for the main conclusion of our consideration, however.

In an approach introduced by Feynman [43] to explain the Josephson effects across a weak link between two superconductors one considers the macroscopic wavefunctions $\psi_{j}=\sqrt{n_{j}} e^{i \phi_{j}}$ on opposite sides of the junction, and treats the weak coupling between them according to $i \hbar \frac{\partial}{\partial t} \psi_{\alpha ; \beta}=\mu_{\alpha ; \beta} \psi_{\alpha ; \beta}+K \psi_{\beta ; \alpha}$, where $K \ll \mu_{j}$ is a phenomenological coupling constant. The resulting differential equations for the number of particles $n_{j}$ occupying the respective macroscopic quantum states and for the corresponding phase difference $\Delta \phi=\phi_{\beta}-\phi_{\alpha}$ become

$$
\begin{aligned}
& \frac{\partial n_{\alpha}}{\partial t}=-\frac{\partial n_{\beta}}{\partial t}=\frac{2 K}{\hbar} \sqrt{n_{\alpha} n_{\beta}} \sin (\Delta \phi), \\
& \frac{\partial \Delta \phi}{\partial t}=\frac{\mu_{\alpha}-\mu_{\beta}}{\hbar}-\frac{K}{\hbar} \frac{n_{\alpha}-n_{\beta}}{\sqrt{n_{\alpha} n_{\beta}}} \cos (\Delta \phi) .
\end{aligned}
$$

For $H_{c, \beta}>H_{c, \alpha}$, the solution of Eq. $1 \mathrm{~b}$ in the weak-coupling limit $K \ll \mu_{\beta}$ is, to a first approximation, $\Delta \phi \approx \Delta \phi_{0}+\omega_{\text {a.c. }}$. with $\omega_{\text {a.c. }} \approx \Delta \mu / \hbar$ and an undetermined constant phase difference $\Delta \phi_{0}$. The resulting variations in $n_{j}(t)$ are very small, i.e., smaller by a factor $K / \Delta \mu$ than their time-averaged values.

\subsection{The d.c. and a.c. Josephson effects}

In the case of identical systems $\left(\Delta \mu=0\right.$ and $n_{\alpha}=n_{\beta}$; see Fig. 2a) a constant $\Delta \phi(t)=$ $\Delta \phi_{0}=\phi_{0, \beta}-\phi_{0, \alpha}$ would represent a constant quasiparticle current $\partial n_{\alpha} / \partial t$ across the weak link (d.c. Josephson effect). As the constants $\phi_{0, j}$ correspond to the in-plane angles $\varphi_{j}$ of the respective transverse magnetic moments that will tend to align in the coupled device, we expect $\Delta \phi_{0}=0$ and therefore $\partial n_{j} / \partial t=0$.

In a device with two magnetic insulators with different critical fields, however, $\Delta \mu \neq 0$ and therefore $\omega_{\text {a.c. }} \neq 0$ (Fig. 2c). As one would expect from the analogy with superconductors and superfluids, the resulting variation $\partial n_{\alpha} / \partial t$ given by Eq. 1a represents an oscillation in time with the field-independent leading frequency $\omega_{\text {a.c. }}$ (a.c. Josephson effect).

The analogous situation in a superconducting Josephson device leads to the appearance of Josephson electrical currents. For the case of connection to an external charge reservoir and fixing $\Delta \mu$ to a constant value, the net charge-carrier density remains constant despite a non-zero $\partial n_{\alpha} / \partial t$, but a measurable electrical current proportional to $\partial n_{\alpha} / \partial t$ flows from the charge reservoir and the attached leads through the whole device [43].

With a junction composed of insulating spin systems it is the external magnetic field that maintains the difference between the chemical potentials at a certain value. This field fully penetrates the whole sample volume, and it therefore entirely "short-circuits" each of the two spin systems separately, thereby keeping the respective total numbers of quasiparticles 

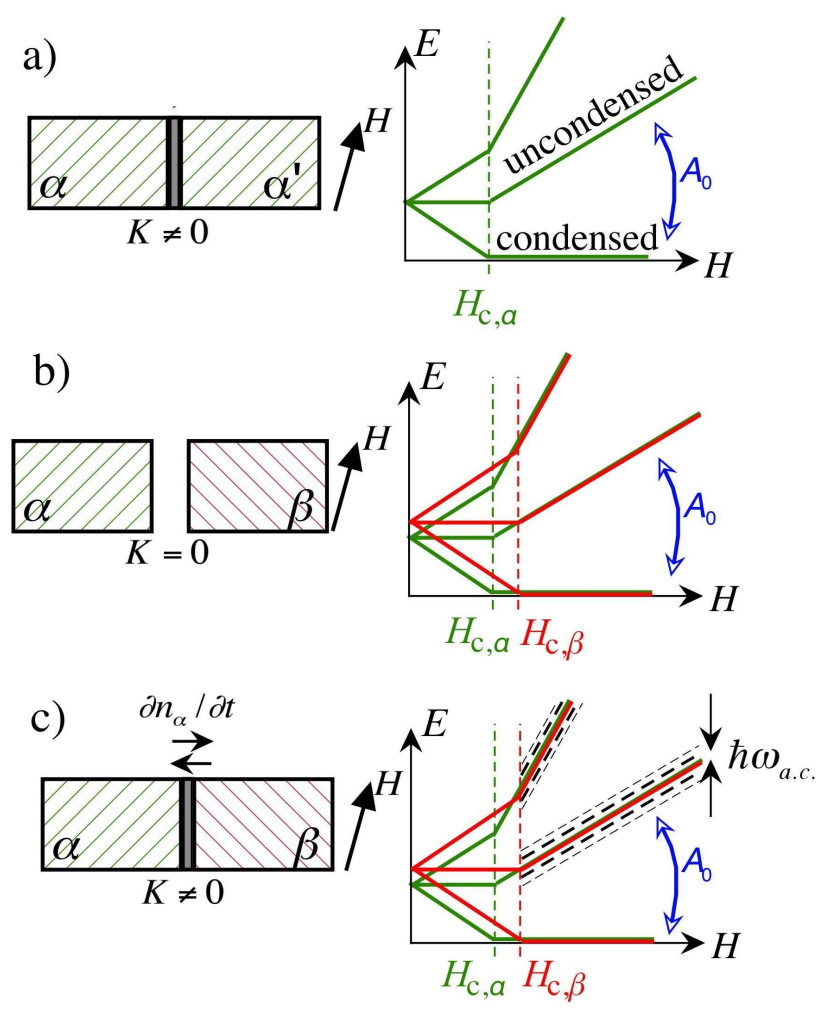

Figure 2: Sketch of the expected energy scheme (right panels) for a) two identical weakly coupled magnetic insulators, b) two uncoupled magnetic insulators with different critical fields, and c) two weakly coupled magnetic insulators with different critical fields (the a.c. Josephson effect), all for $H>H_{c, j}$ and measured relative to the respective ground-state energies. In the case c), the ESR-active mode $A_{0}$ should split by a detectable amount $\hbar \omega_{\text {a.c }}$ (see the text). Arrows in the left panels symbolize the quasiparticle currents related to $\partial n_{\alpha} / \partial t$ that can be ascribed to the spacer region (see the text).

$n_{j}$ constant. As a consequence, there is no directional macroscopic quasiparticle current flowing within the two individual branches on both sides of the device. One can think of the magnetic field as a quasiparticle source (or sink) that replaces (or removes) those quasiparticles that are crossing the weak link. It is only by virtue of the phase difference $\Delta \phi$ between the two materials that a quasiparticle current can still be formally ascribed to the magnetically inert spacer region (see Fig. 2c), but it may be impossible to detect it directly in an experiment.

\subsection{Experimental manifestation of the a.c. Josephson effect}

To second-order approximation ( $K \neq 0$ with $H_{c, \alpha} \neq H_{c, \beta}$ and therefore $n_{\alpha} \neq n_{\beta}$ ), Eq. $1 \mathrm{~b}$ denotes a narrowband frequency modulation of the phases

$$
\phi_{\alpha ; \beta}(t) \approx \phi_{0, \alpha ; \beta}-\frac{\mu_{\alpha ; \beta}}{\hbar} t-\frac{K}{\Delta \mu} \sqrt{\frac{n_{\beta ; \alpha}}{n_{\alpha ; \beta}}} \sin \left(\omega_{\text {a.c. }} t+\Delta \phi_{0}\right),
$$

with a modulation frequency $\omega_{\text {a.c. }}$ and a modulation index of the order of $K / \Delta \mu$. This modulation of $\phi_{j}(t)$ has profound consequences on the macroscopic wavefunctions $\psi_{j}=$ 
$\sqrt{n_{j}} e^{i \phi_{j}}$ describing the two condensates, because equally spaced sidebands should appear in the energy spectrum of the coupled device. This dynamic effect should manifest itself in a corresponding splitting of all energies that are associated with transitions from the (condensed) ground state to (uncondensed) excited states (see Fig. 2c). Such a splitting is absent for two uncoupled magnetic insulators with different critical fields (Fig. 2b) and, of course, also in a scenario where no macroscopic phase coherence is present at all. Therefore a successful experiment in a coupled system that can probe the occurrence of sidebands with separation $\hbar \omega_{\text {a.c. }}$ would represent very strong experimental support for the existence of a state with macroscopic phase coherence.

To be more specific, a high-resolution electron-spin resonance (ESR) measurement of the transitions between the ground state considered here, and the nearest excited triplet states (referred to as $A_{0}$ in Ref. [44] and $E_{0}(Q)$ in Ref. [45]), or a one-magnon Raman experiment on the so-called $E_{-}(Q)$ mode [46], should reveal a characteristic splitting of the corresponding modes by $\hbar \omega_{\text {a.c. }}$ which is related to the a.c. Josephson effect (see Fig. 2c). This is a central result of this work, and we will discuss in the following whether or not the proposed a.c. Josephson effect can be observed in a device with realistic material parameters.

\section{Constraints in real systems}

\subsection{The lifetime of phase coherence}

We may expect a successful experiment only if the separation $\hbar \omega_{\text {a.c. }}$ between associated sidebands in the energy spectrum is comparable to or larger than their linewidth which can be related to the inverse lifetime $\tau_{p c}^{-1}$ of the phase coherence and to the inverse lifetime $\tau_{q p}^{-1}$ of the magnetic quasiparticles involved, i.e., $\omega_{\text {a.c. }} \gtrsim \max \left(\tau_{p c}^{-1}, \tau_{q p}^{-1}\right)$. The lifetime $\tau_{p c}$ has, to the best of our knowledge, not yet been estimated for triplon BECs, but we may initially compare it to $\tau_{q p}$, i.e., that of the $S=1$ quasiparticles. Processes of scattering on magnons, phonons or impurities can be important limiting factors. As long as the external magnetic field remains in the expected stability region of the condensate (i.e., close to $H_{c}$ where $n \ll 1[10,47])$, however, there is no fundamental principle that would restrict this lifetime to inaccessibly short time scales at low enough temperatures. Corresponding values for the quasiparticle lifetime $\tau_{q p}$ ranging from $>10^{-11} \mathrm{~s}$ up to $\approx 5 \times 10^{-11} \mathrm{~s}$ can be inferred from precise inelastic neutron-scattering measurements of the magnon linewidth in the spindimerized compounds $\mathrm{TlCuCl}_{3}$ [48] and $\left(\mathrm{C}_{4} \mathrm{H}_{1} 2 \mathrm{~N}_{2}\right) \mathrm{Cu}_{2} \mathrm{Cl}_{6}$ [49], respectively, taken at low temperatures for quasiparticles that are not part of the condensate, and from the ESR linewidth of the $A_{0}$ mode observed in $\mathrm{TlCuCl}_{3}[44]$.

It is conceivable that the actual lifetime $\tau_{p c}$ of phase coherence in the BEC is even considerably longer than that of its constituting particles $\tau_{q p}$, e.g., thanks to the formation of energetic barriers of topological origin that may lead to a strong suppression of dissipation, i.e., to the occurrence of true spin superfluidity [37]. It is worth mentioning here that the a.c. Josephson oscillations that have been seen in an experiment on an exciton-polariton Josephson junction were clearly observable although the oscillation frequency $\left(\omega_{\text {a.c. }} \approx 4 \times\right.$ $\left.10^{11} \mathrm{~s}^{-1}\right)$ was very near the inverse lifetime of the respective bosonic quasiparticles $\left(\tau_{q p}^{-1} \approx\right.$ 
$3 \times 10^{11} \mathrm{~s}^{-1}$ ) [32], but still much larger than the inverse of the surprisingly long measured phase-coherence time $\left(\tau_{p c}^{-1} \approx 7 \times 10^{9} \mathrm{~s}^{-1}\right.$ [50], i.e., $\left.\tau_{p c} \gg \tau_{q p}\right)$. To be on the safe side we conservatively choose $\omega_{\text {a.c. }} \approx 10^{11} \mathrm{~s}^{-1}>\tau_{q p}^{-1}$ for our proposed experiment, which corresponds to $\mu_{0}\left(H_{c, \beta}-H_{c, \alpha}\right) \approx 0.5 \mathrm{~T}$.

\subsection{Violated axial symmetry}

So far we have considered only perfectly axially symmetric materials. Any violation of axial symmetry of the magnetic exchange interaction leads to the occurrence of an anisotropy gap $\Delta$ in the energy spectrum $E(k)$ [55], thereby lifting the Goldstone linearity for $k \rightarrow$ 0 , fixing the constants $\phi_{0, j}$ to specific values $[3,9]$ and leading to well-defined magnetic structures with in-plane angles $\varphi_{j}$ that are locked to the crystal lattice $[39,40]$. This gap has been estimated to be $\Delta(H)=\sqrt{8 \tilde{\gamma} \mu(H)}$, where $\tilde{\gamma}>0$ is a measure for the exchangeinteraction anisotropy in the plane perpendicular to $H$ [55]. For experimental energies larger than $\Delta$, however, a quasi-linear Goldstone mode is recovered [6], the effects of axial anisotropy are expected to be smeared out on short enough time scales, and we can ascribe an upper limit $\tau_{\Delta}=\hbar / \Delta$ to the lifetime of the phase coherence [9] so that we must additionally require $\omega_{\text {a.c. }}>\tau_{\Delta}^{-1}$.

\subsection{The weak link}

In a real experiment, $\Delta \mu=g \mu_{0} \mu_{B}\left(H_{c, \beta}-H_{c, \alpha}\right)$ and the coupling constant $K$ are determined by the junction properties. The dimensions of the weak link should not significantly exceed the healing length of the condensate (the analogue to the coherence length in superconductors). For weakly interacting Bose gases, this healing length is $\xi=\hbar / \sqrt{2 n v_{0} m^{*}}$ (where $m *$ is the effective mass of the quasiparticles) [51] and it diverges as the temperature is increased towards the Bose-Einstein condensation temperature where $n$ vanishes.

The simplest version of such a weak link may consist of a slit that is narrow enough to allow for a small but finite magnetic coupling and therefore for a tunneling of magnetic quasiparticles. Such a junction might also be manufactured by the layered growth of a magnetic insulator $\left(\alpha\right.$, with $\left.H_{c, \alpha}\right)$ containing a certain species of magnetic ions forming spin$1 / 2$ dimers, followed by one or a few layers of an isostructural compound with nonmagnetic ions, and by further adjacent layers of another isostructural magnetic insulator $(\beta$, with $\left.H_{c, \beta} \neq H_{c, \alpha}\right)$.

\subsection{A realistic toy model: $(\mathrm{Ba}, \mathrm{Sr})_{3} \mathrm{Cr}_{2} \mathrm{O}_{8}$}

To obtain numerical values, we consider a model device composed of $\mathrm{Ba}_{3} \mathrm{Cr}_{2} \mathrm{O}_{8}(\alpha)$ with $\mu_{0} H_{c, \alpha} \approx 12 \mathrm{~T}$ [40], separated by nonmagnetic isostructural $\mathrm{Ba}_{3} \mathrm{~V}_{2} \mathrm{O}_{8}$ [52] from a compound $\mathrm{Ba}_{3-x} \mathrm{Sr}_{x} \mathrm{Cr}_{2} \mathrm{O}_{8}(\beta)[53]^{2}$ with $x$ chosen in such a way as to achieve a larger $\mu_{0} H_{c, \beta}$, but still much smaller than the value $\mu_{0} H_{c 2}=30 \mathrm{~T}$ for fully stoichiometric $\mathrm{Sr}_{3} \mathrm{Cr}_{2} \mathrm{O}_{8}$ with $x=$ 1 [53]. If we achieve a $\mu_{0} H_{c, \beta} \approx 12.5 \mathrm{~T}$, the expected characteristic frequency becomes $\omega_{\text {a.c. }} \approx 10^{11} \mathrm{~s}^{-1}$. With an external magnetic field $\mu_{0} H \approx 13 \mathrm{~T}$, and taking $v_{0} / k_{B} \approx 8.7 \mathrm{~K}$

\footnotetext{
${ }^{2}$ We have already verified the existence of the solid-solution series $\mathrm{Ba}_{3-x} \mathrm{Sr}_{x} \mathrm{Cr}_{2} \mathrm{O}_{8}$ for polycrystalline samples, but we have no further information about their magnetic properties.
} 
with $g \approx 2[54]$ we have $n_{\alpha} \approx 0.15$ and $n_{\beta} \approx 0.08$. The choice of a small $H-H_{c, j}$ (i.e., $\left.\mu_{j} \ll v_{0}\right)$ is primarily necessary to fulfill the condition $n_{j} \ll 1$, but it may not be crucial for observing the essence of the predicted effect. With $m^{*} \approx 1.5 \times 10^{-27} \mathrm{~kg}$ and a mean distance $d \approx 0.6 \mathrm{~nm}$ between the dimers $[40,54]$, the resulting values for the healing lengths at $T=0$ are small $\left(\xi_{\alpha} \approx 0.4 \mathrm{~nm}\right.$ and $\left.\xi_{\beta} \approx 0.6 \mathrm{~nm} \approx d\right)$, but a very narrow slit or a weak link composed of one or only a few unit cells of the nonmagnetic compound could be adequate. If still larger values of $\xi_{j}$ are required, $n_{j}$ can be diminished by further reducing $\Delta \mu$ and $\mu_{\beta}$. Alternatively, the experiment can be performed near the condensation temperature as has been done for superfluid ${ }^{4} \mathrm{He}$ to make the a.c. Josephson effect observable [27], possibly at the cost of reducing the lifetime $\tau_{q p}$ of the triplon quasiparticles.

With the material parameters used for the experiment described above and $\omega_{\text {a.c. }} \approx$ $10^{11} \mathrm{~s}^{-1}, \tilde{\gamma}$ should be below $\approx 4 \mu \mathrm{eV}$. The exchange-interaction anisotropy has been estimated to be $\tilde{\gamma} \approx 16-30 \mu \mathrm{eV}$ in $\mathrm{TlCuCl}_{3}[9,56]$, but to only be $\tilde{\gamma} \approx 1 \mu \mathrm{eV}<4 \mu \mathrm{eV}$ in $\mathrm{BaCuSi}_{2} \mathrm{O}_{6}$ [57] and particularly in $\mathrm{Ba}_{3} \mathrm{Cr}_{2} \mathrm{O}_{8}$ [40], so the proposed device would fulfill all of the requirements listed above.

\section{Concluding remarks}

To conclude these numerical estimates we want to state that other material properties, such as the presence of a strong Dzyaloshinski-Moriya interaction that we have not considered here, may still inhibit the formation of a phase-coherent condensate [55] and therefore make the observation of the a.c. Josephson effect impossible. However, we classify such factors as not intrinsic to the problem, and a proper choice of compounds with suitable material parameters should make the generation and the observation of the a.c. Josephson effect in magnetic insulators feasible in principle. Although we have chosen dimerized spin systems operating near their lower critical fields as a model for the present consideration, all of the above arguments should also hold near the respective saturation fields as a consequence of the particle-hole symmetry of the problem [4], and for all other kinds of insulating spin systems that are supposed to show a field-induced BEC of magnetic quasiparticles.

It is conceivable that Josephson-like phenomena even naturally occur in certain quantum magnets, e.g., in a system showing an intrinsic modulation of the chemical potential due to magnetically inequivalent planes hosting the triplon condensate. A situation in which the average condensate density strongly varies along the $c$-axis, as a consequence of different intra-dimer exchange couplings and resulting different energy gaps within alternating layers, has been reported to occur in $\mathrm{BaCuSi}_{2} \mathrm{O}_{8}$ [58-60].

We finally mention here an interesting analogy between the case of an ESR measurement on weakly coupled magnetic insulators as discussed above, and corresponding microwave stimulated experiments on ferromagnetic films which are separated by normal-metal spacers [61]. In both cases, there exists a dynamic coupling between two different materials through a magnetically inert spacer layer, thereby altering the dynamics in both systems as compared to the uncoupled situation.

In summary, we suggest that two weakly linked magnetic insulators with different critical fields $H_{c, j}$ that are placed into a suitably chosen external magnetic field should show an 
altered energy spectrum as compared to the uncoupled limit, with additional sidebands that are separated by $\hbar \omega_{\text {a.c. }}=g \mu_{B} \mu_{0}\left(H_{c, \beta}-H_{c, \alpha}\right)$. These sidebands are a manifestation of the a.c. Josephson effect and can be tested in, for example, a high-resolution ESR experiment. Assuming realistic material parameters and considering several constraints, we conclude that this effect can indeed take place in a real system. A corresponding successful experiment would represent very strong experimental support for the existence of a state with macroscopic phase coherence.

\section{Acknowlegdements}

We thank A. Rosch, J. Roos, T. Giamarchi, M. Matsumoto and A. Rakhimov for stimulating discussions. This work was supported by the Swiss National Foundation Grant. No. 21-126411.

\section{References}

[1] T. Matsubara and H. Matsuda, Prog. Theor. Phys. 16 (1956) 569.

[2] E. G. Batyev and L. S. Braginskii, Sov. Phys. JETP 60 (1984) 781.

[3] I. Affleck, Phys. Rev. B 43 (1991) 3215.

[4] T. Giamarchi and A. M. Tsvelik, Phys. Rev. B 59 (1999) 11398.

[5] T. Nikuni, M. Oshikawa, A. Oosawa and H. Tanaka, Phys. Rev. Lett. 84 (2000) 5868.

[6] Ch. Rüegg, N. Cavadini, A. Furrer, H.-U. Güdel, K. Krämer, H. Mutka, A. Wildes, K. Habicht and P. Vorderwisch, Nature 423 (2003) 62.

[7] T. Giamarchi, Ch. Rüegg and O. Tcherniyshyov, Nature Phys. 4 (2008) 198.

[8] M. Matsumoto, B. Normand, T. M. Rice and M. Sigrist, Phys. Rev. B 69 (2004) 054423.

[9] R. Dell'Amore, A. Schilling and K. Krämer, Phys. Rev. B 79 (2009) 014438.

[10] A. Rakhimov, E. Y. Sherman and C. K. Kim, Phys. Rev. B 81 (2010) 020407.

[11] V. M. Kalita and V. M. Loktev, JETP Lett. 91 (2010) 183.

[12] Y. M. Bunkov and G. E. Volovik, arXiv:1003.4889v1.

[13] D. L. Mills, Phys. Rev. Lett. 98 (2007) 039701.

[14] J. F. Allen and A. D. Misener, Nature 141 (1938) 75.

[15] P. Kapitza, Nature 141 (1938) 74.

[16] M. H. Anderson, J. R. Ensher, M. R. Matthews, C. E. Wieman and E. A. Cornell, Science 269 (1995) 198.

[17] S. O. Demokritov, V. E. Demidov, O. Dzyapko, G. A. Melkov, A. A. Serga, B. Hillebrands and A. N. Slavin, Nature 443 (2006) 430.

[18] V. E. Demidov, O. Dzyapko, S. O. Demokritov, G. A. Melkov and A. N. Slavin, Phys. Rev. Lett. 100 (2008) 047205.

[19] H. Deng, G. Weihs, C. Santori, J. Bloch and Y. Yamamoto, Science 298 (2002) 199.

[20] J. Kasprazak, M. Richard, S. Kundermann, A. Baas, P. Jeambrun, J. M. J. Keeling, F. M. Marchetti, M. H. Szymanska, R. André, J. L. Staehli, V. Savona, P. B. Littlewood, B. Deveaud and L. S. Dang, Nature 443 (2006) 409.

[21] E. Hoskinson, Y. Sato and R. Packard, Phys. Rev. B 74 (2006) 100509.

[22] R.W. Simmonds, A. Marchenkov, E. Hoskinson, J. C. Davis and R. E. Packard, Nature 412 (2001) 55.

[23] M. R. Andrews, C. G. Townsend, H.-J. Miesner, D. S. Durfee, D. M. Kurn and W. Ketterle, Science 275 (1997) 637.

[24] H. E. Hall and W. F. Vinen, Proceedings of the Royal Society A 238 (1956) 204.

[25] M. R. Matthews, B. P. Anderson, P. C. Haljan, D. S. Hall, C. E. Wieman and E. A. Cornell, Phys. Rev. Lett. 83 (1999) 2498. 
[26] K. G. Lagoudakis, M. Wouters, M. Richard, A. Baas, I. Carusotto, R. André, L. S. Dang and B. Deveaud-Plédran, Nature Phys. 4 (2008) 706.

[27] K. Sukhatme, Y. Mukharsky, T. Chui and D. Pearson, Nature 411 (2001) 280.

[28] A. S. Borovik-Romanov, Y. M. Bun'kov, A. deVaard, V. V. Dmitriev, V. Matrotsieva, Y. M. Mukharskii and D. A. Sergatskov, JETP Lett. 47 (1988) 478.

[29] S. V. Pereverzev, A. Loshak, S. Backhaus, J. C. Davis and R. E. Packard, Nature 388 (1997) 449.

[30] B. P. Anderson and M. A. Kasevich, Science 282 (1998) 1686.

[31] S. Levy, E. Lahoud, I. Shomroni and J. Steinhauer, Nature 449 (2007) 579.

[32] K. G. Lagoudakis, B. Pietka, M. Wouters, R. André and B. Deveaud-Plédran, Phys. Rev. Lett. 105 (2010) 120403.

[33] V. P. Peshkov, J. Physics (Moscow) 8 (1944) 381.

[34] A. S. Borovik-Romanov, Y. M. Bun'kov, V. V. Dmitriev and Y. M. Mukharskii, JETP Lett. 40 (1984) 1033.

[35] C. Raman, M. Köhl, R. Onofrio, D. S. Durfee, C. E. Kuklewicz, Z. Hadzibabic and W. Ketterle, Phys. Rev. Lett. 83 (1999) 2502.

[36] A. Amo, D. Sanvitto, F. P. Laussy, D. Ballarini, E. del Valle, M. D. Martin, A. Lemaître, J. Bloch, D. N. Krizhanovskii, M. S. Skolnick, C. Tejedor and Viña L., Nature 457 (2009) 291.

[37] E. B. Sonin, Adv. Phys. 59 (2010) 181.

[38] F. S. Nogueira and K.-H. Bennemann, Europhys. Lett. 67 (2004) 620.

[39] H. Tanaka, A. Oosawa, T. Kato, H. Uekusa, Y. Ohashi, K. Kakurai and A. Hoser A., J. Phys. Soc. Jpn. 70 (2001) 939.

[40] M. Kofu, H. Ueda, H. Nojiri, Y. Oshima, T. Zenmoto, K. C. Rule, S. Gerischer, B. Lake, C. D. Batista, Y. Ueda and S.-H. Lee, Phys. Rev. Lett. 102 (2009) 177204.

[41] L. P. Pitaevskii and S. Stringari, Bose-Einstein Condensation, Oxford Science Publications, 2003.

[42] R. Ozeri, N. Katz, J. Steinhauer and N. Davidson, Rev. Mod. Phys. 77 (2005) 187.

[43] R. P. Feynman, R. B. Leighton and M. Sands, The Feynman Lectures on Physics Vol. III, AddisonWesley, 1965.

[44] S. Kimura, M. Hagiwara, H. Tanaka, A. Kolezhuk and K. Kindo, J. Magn. Magn. Mater. 310 (2007) 1218.

[45] M. Matsumoto, T. Shoji and M. Koga M., J. Phys. Soc. Jpn. 77 (2008) 074712.

[46] H. Kuroe, K. Kusakabe, A. Oosawa, T. Sekine, F. Yamada, H. Tanaka and M. Matsumoto, Phys. Rev. B 77 (2008) 134420.

[47] A. Rakhimov, S. Mardonov, and E. Y. Sherman, Ann. Phys. (NY) 326 (2011) 2499.

[48] Ch. Rüegg, B. Normand, M. Matsumoto, C. Niedermayer, A. Furrer, K. W. Krämer, H.-U. Güdel, P. Bourges, Y. Sidis and H. Mutka, Phys. Rev. Lett. 95 (2005) 267201.

[49] M. B. Stone, C. Broholm, D. H. Reich, P. Schiffer, O. Tchernyshyov, P. Vorderwisch and N. Harrison, New J. Phys. 9 (2007) 31.

[50] A. P. D. Love, D. N. Krizhanovskii, D. M. Whittaker, R. Bouchekioua, D. Sanvitto, S. A. Rizeiqi, R. Bradley, M. S. Skolnick, P. R. Eastham, R. André and L. S. Dang, Phys. Rev. Lett. 101 (2008) 067404.

[51] N. N. Bogoliubov, J. Phys. (USSR) 11 (1947) 23.

[52] P. Süsse and M. J. Buerger, Z. Kristallogr. 131 (1970) 161.

[53] A. A. Aczel, Y. Kohama, C. Marcenat, F. Weickert, M. Jaime, O. E. Ayala-Valenzuela, R. D. McDonald, S. D. Selesnic, H. A. Dabkowska and G. M. Luke, Phys. Rev. Lett. 103 (2009) 207203.

[54] T. Dodds, B.-J. Yang and Y. B. Kim, Phys. Rev. B 81 (2010) 054412.

[55] J. Sirker, A. Weisse and O. P. Sushkov, J. Phys. Soc. Jpn. 74 (2005) Suppl. 129.

[56] A. K. Kolezhuk, V. N. Glazkov, H. Tanaka and A. Oosawa A., Phys. Rev. B 70 (2004) 020403.

[57] S. E. Sebastian, P. Tanedo, P. A. Goddard, S.-C. Lee, A. Wilson, S. Kim, S. Cox, R. D. McDonald, S. Hill, N. Harrison, C. D. Batista and I. R. Fisher, Phys. Rev. B 74 (2006) 180401.

[58] Ch. Rüegg, D. F. McMorrow, B. Normand, H. M. Rønnow, S. E. Sebastian, I. R. Fisher, C. D. Batista, S. N. Gvasaliya, Ch. Niedermayer, and J. Stahn, Phys. Rev. Lett. 98 (2007) 017202.

[59] S. Krämer, R. Stern, M. Horvatić, C. Berthier, T. Kimura, and I. R. Fisher, Phys. Rev. B 76 (2007) 
100406(R).

[60] N. Laflorencie and F. Mila, Phys. Rev. Lett. 102 (2009) 060602.

[61] B. Heinrich, Y. Tserkovnyak, G. Woltersdorf, A. Brataas, R. Urban and G. E. W. Bauer, Phys. Rev. Lett. 90 (2003) 187601. 